\title{
Key Technology for On-line Detection of Partial Discharge in High Voltage Switchgear
}

\author{
Pan Jianqiao, Wu Di, Jin Chaohui, Luan Yinbing, Xu Liang \\ State Grid Zhejiang Pinghu Power Supply Limited Company, Zhejiang Pinghu, 314200
}

Keywords: High voltage switchgear, partial discharge, online detection, key technology

\begin{abstract}
The article focuses on the partial discharge phenomenon of high-voltage switchgear. On the basis of grasping the significance of online detection, the paper analyzes the type of partial discharge of high-voltage switchgear and the specific detection methods. Based on the data obtained from the research, the planning and construction of the overall structure of the online detection system and the implementation path are repositioned. From a certain point of view, the stability and reliability of the online detection system plays an important role in promoting the improvement and optimization of the entire power grid structure.
\end{abstract}

\section{Introduction}

As a basic equipment type in the power system, the high-voltage switchgear has an indelible role and position in the entire electrical equipment structure. Its operational safety and reliability are related to the stability and continuity of the entire power grid. If the operation of the high-voltage switchgear is abnormal, such as internal insulation degradation, defective or poorly connected parts of the conductive connection, it is very likely to damage the operating rules of the power grid and cause serious safety accidents ${ }^{[1]}$.Through the investigation and research on relevant domestic data, as of the end of 2017, the national switchgear operation accidents occurred frequently, and the incidence of insulation accidents was mostly, accounting for $40 \%$ of the total number of failures. In many insulation accidents, flashover accidents, the statistics are nearly $80 \%$. However, most of the partial discharge phenomenon is lurking in the initial stage of the accident. The online detection technology can realize the on-line detection of the partial discharge phenomenon, and systematically evaluate and judge the specific insulation state inside the high-voltage switchgear according to the test results.

\section{Comprehensive Explanation of the Phenomenon of Partial Discharge}

The so-called partial discharge is essentially a discharge phenomenon formed by the power plant insulator as a medium and through the super strong action of the power plant in a local range. However, this discharge phenomenon is limited to some extent by the shorting of the insulating cloth between the conductors without forming a conductive path. ${ }^{[2]}$. According to the investigation, liquid insulating bubbles, solid insulating holes, different characteristic insulating layers and sharp edges of metal electrodes are all frequent parts of partial discharge phenomena. Generally, air gaps are common. When the partial discharge reaches a certain level, it is easy to directly affect the medium and weaken the internal insulation strength of the system. Therefore, when designing the insulation part of high-voltage power equipment, it should be straight line on the premise of ensuring voltage running time and running strength, as far as possible, avoid the occurrence of strong partial discharge in the insulation structure. The detection of the operating power equipment should be further enhanced. Once the partial discharge is found to exceed the standard, the equipment operation should be terminated immediately, and the equipment should be repaired or replaced in time. ${ }^{[3]}$.

\section{Significance of Partial Discharge Monitoring of High Voltage Switchgear}

From a conventional point of view, for the detection of internal faults in high-voltage switchgear, it is necessary to ensure that the system is in a power outage state. At present, the implementation 
strength and scope of online detection are relatively small. Affected by various factors, the inspection and maintenance of high-voltage switchgear cannot be carried out with periodic power failure maintenance standards, which cannot touch the internal components of the switchgear during the monitoring process. At the same time, some components have problems such as out of control and disrepair, to some extent. It is not conducive to the normal operation of the high-voltage switchgear, which poses a great threat to the safety and stability of the entire power supply system. Moreover, during different periodic inspections, the interior is still in the monitoring blind spot, and it is impossible to predict and evaluate its operating status, and it is difficult to predict the possible safety accidents ${ }^{[4]}$. Once a serious failure occurs in the equipment and it cannot be detected and handled in the first time, the scope of the damage will be infinitely expanded, which will easily damage the working environment and power supply safety. The components of the switchgear are all installed inside the switchgear. The patrol personnel cannot observe the inside of the switchgear, so the online monitoring system of the high-voltage switchgear is particularly important.

\section{High-Voltage Switchgear Partial Discharge Online Monitoring}

\subsection{Partial Discharge type}

\subsubsection{Corona Discharge}

Generally, corona discharge occurs mostly around a high-voltage conductor surrounded by a gas, such as a high-voltage transformer or a high-voltage transmission line, etc., since the high-voltage terminal is exposed to the gas layer for a long time, causing frequent occurrence of corona discharge. In the corona discharge process, the characteristics of electrode non-uniformity are the main basis for judging whether there is discharge behavior, and it is also a self-sustained discharge form unique to extremely uneven electric fields. In addition, the corona start voltage produces different amplitude changes under different external conditions, such as electrode shape, electrode distance, gas density, air humidity, applied voltage or electrode flow speed, etc. ${ }^{[5]}$.

\subsubsection{Discharge Along the Surface}

Unlike corona discharge, creeping discharge often occurs on the surface of the dielectric medium. It can be listed as a special gas discharge in terms of type. The parts with higher frequency are mostly the position of power cable and insulated sleeve end. If the intensity of the electric field at the edge of the motor is higher than the intensity of the electric field inside the medium, and the dielectric has a lower breakdown voltage along the surface, it is easy to cause creeping discharge on the surface of the dielectric. According to the formation process of creeping discharge voltage, voltage waveform, air quality, climatic conditions, power plant distribution and surface state of the medium have a direct influence on the creeping discharge voltage, and also exhibit the instability of creeping discharge from the side feature.

\subsubsection{Internal Discharge}

Internal discharge mainly refers to the discharge phenomenon generated inside the solid insulating medium. Usually, in the process of production and processing of insulating media, the production materials and production processes are not up to standard or there are problems such as quality defects. This drawback also becomes a direct cause of defects in the insulating medium, among which more common problems, such as Mix a small amount of air or impurities. If a high-intensity voltage is applied to the insulating medium, local breakdown or repetitive breakdown is highly likely to occur, and from the causes of internal discharge and various conditions, it is generally related to the characteristics of the medium itself, the shape of the defect, and the air gap. The size and type of gas gap are closely related ${ }^{[6]}$.

\subsubsection{Suspension Potential Discharge}

The definition of the suspension potential discharge is mainly based on the relevant conductor parts in the high-voltage equipment, the analysis of the design defects around the part structure, or the 
components are affected by the characteristics of contact, connectivity, etc., and are placed between the high and low voltage electrodes, and according to The impedance of the position is discharged compared to the obtained partial pressure, which is referred to as the floating potential for the ground potential on the conductor member. When the floating potential is present in the conductor, the surrounding electrodes can be rapidly aggregated, but it is easy to cause serious damage to the formation process of the insulating medium.

\subsection{Types of Partial Discharge of Switchgear and Cables}

There are two main types of partial discharges in switch cabinets and cables: one is surface discharge and the other is internal discharge. Both surface discharge and internal discharge can cause insulation failure of the device and even catastrophic consequences.

On the one hand, environmental conditions affect.

\subsubsection{The Temperature is too High or too Low}

If the operating temperature of the high-voltage switchgear is too high, it will accelerate the aging of the surface insulation material and damage the insulation of the surface layer of the medium. On the contrary, when the ambient temperature is low, it will easily lead to a sharp drop in the robustness of the switch-insulated porcelain bottle and the vacuum cylinder, and it is easy to cause a shattering phenomenon during the opening and closing of the electric gate. In severe cases, the switch has a short circuit or even an explosion.

\subsubsection{Insulation Surface Area Contamination}

Dust and fly ash are two common types of dirt on the insulating surface. The dust is mostly attached to indoor equipment. The long-term accumulation of dirt not only affects the pressure resistance of the insulation power frequency, but also weakens the impact of insulation to some extent ${ }^{[7]}$.

\subsubsection{Wet}

Under normal circumstances, if there is a large amount of dirt in the insulating surface layer, but the environment is relatively dry, the influence on the resistance is small, and the possibility of flashover is small, and when the insulating medium is damp, a thin layer is formed. The conductive liquid film gradually decomposes the electrolyte of the dirty layer, rapidly reducing the dielectric strength of the electrical equipment, and more serious problems such as flashover.

On the other hand, the device's cause.

Equipment Manufacturing and Process Defects

For the judgment of the overall quality and pressure level of the switchgear, the manufacturing quality and assembly quality are usually the main indicators. However, in most cases, the withstand voltage test is applicable to some components in the switchgear, but it cannot be applied to the quality inspection process of the entire switchgear. The main reason is that the assembly quality is not up to standard. For example, there are irregularities in the tightness of the screw. After the screw is tightened, it is slightly longer than the nut; after special treatment of the insulating porcelain sleeve, it is not only unfavorable for the control of the insulation distance, but also easy to cause the electric field to be too concentrated; the quality of the supporting porcelain or porcelain sleeve is not up to standard, and the stability level is low. It is unable to withstand the impact of short-circuit current, and it is easy to deepen and expand the impact degree and scope of the accident.

The Contact Capacity is Insufficient or the Contact is Poor, and the Heat Causes the Switch Cabinet to Catch Fire

For the formation of faults such as insufficient contact capacity or poor contact, it is not only easy to be too high local temperature, but also easily cause damage to the current-carrying portion, causing flashing to the ground or phase, thereby causing the formation of the insulation flashover.

The Creepage Distance and the Air Gap are Insufficient.

One of the important reasons for the frequent occurrence of damage to the switchgear insulator is the lack of creepage and air gap. Especially in the production and application process of handcart 
cabinets, the relevant manufacturers have purposefully shortened the distance between the isolation plugs, circuit breakers and grounding in the hand cabinet, and adopted the unreasonable insulation strength to achieve the cabinet size of effectively shortened.

\subsubsection{TEV Detection Method}

When a partial discharge phenomenon occurs with a high-voltage electrical device, a large amount of discharged electricity is accumulated in the grounded metal portion adjacent to the discharge point, and propagates in various directions in the form of electromagnetic waves. To some extent, the discharge power in the internal discharge is mostly grounded. The shielding surface is concentrated, so the detection of the discharge signal needs to avoid the continuous shielding layer. However, from the practical point of view, field breakage often occurs at the joints of the gaskets, the insulation parts, and the terminals of the cable insulation, which affects the continuity and effectiveness of the entire shielding layer, so that the high-frequency electromagnetic signals are transmitted to the outer layer of the device.

The electromagnetic wave generated by the discharge propagates through the joint of the metal box or the gasket of the gas insulated switch, and simultaneously generates a transient voltage, which is transmitted to the ground through the outer surface of the metal case of the device ${ }^{[8]}$. These voltage pulses are transient to ground voltages, referred to as TEV. The principle of TEV detection is shown in Figure 1.

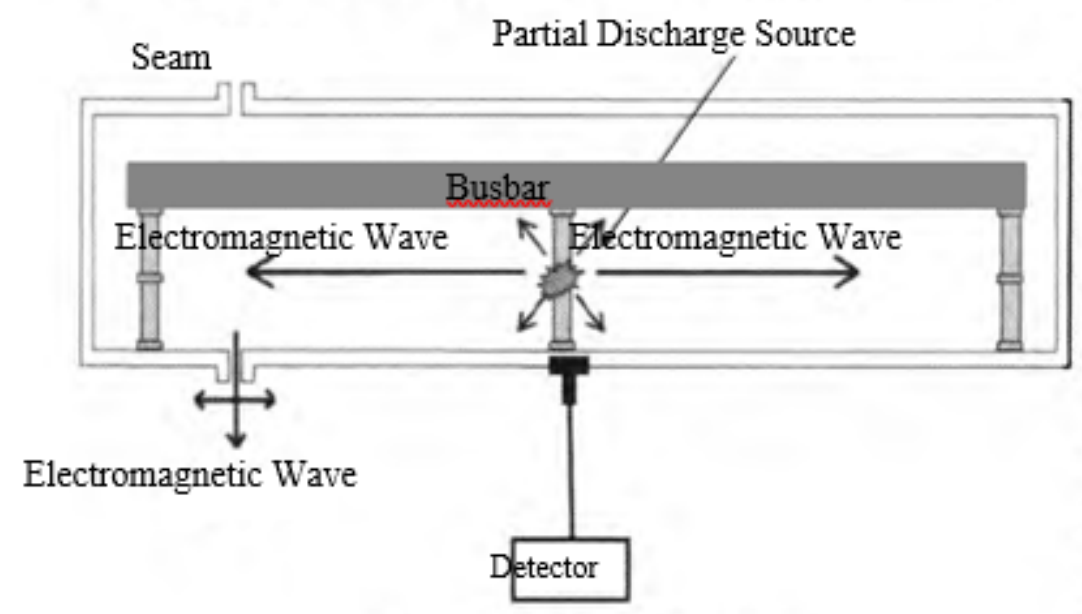

Figure 1

\subsubsection{PDLI Positioning Principle}

According to relevant research and analysis, the size of the TEV signal generated during partial discharge directly affects the intensity of the discharge and the proximity of the discharge point to some extent, and can be detected by a professional detector. For example, the use of the reading (dB) represents the extent of partial discharge activity. Figure 2 shows the partial discharge positioning diagram. Through the effective detection of the TVE signal, the partial discharge condition of the equipment in the switchgear can be quantitatively tested, and the local discharge power can be performed by the time difference of the same discharge source to position different detectors ${ }^{[9]}$. The PDLI determines the position of the discharge source by detecting the sequence of the partial discharge high-frequency pulse reaching the two detectors through two capacitive coupling detectors. The schematic diagram of the on-site positioning is shown in Fig. 2. 


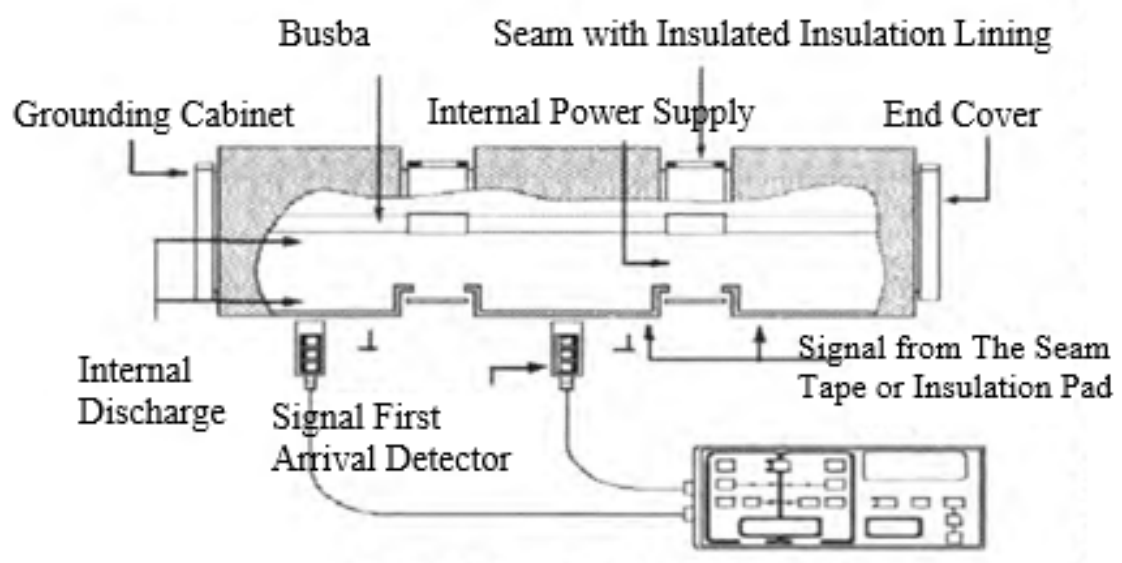

Figure 2

\section{Conclusion}

In summary, in today's rapid development of the electric power industry, the safety and stability of the operation of the power grid system play an important role, and online detection is an important way to judge the partial discharge phenomenon of the high-voltage switch, and is closely related to the safety of the entire power grid system ${ }^{[10]}$. The online detection technology can not only accurately locate the position of the local power supply head, but also greatly improve the overall efficiency of the maintenance work, effectively shorten the time spent on power failure maintenance, avoid excessive loss and waste of limited power energy, and thus ensure the ultimate economic benefits of the power system.

\section{References}

[1] Zaiyu Cui, Changyuan Jiang, Jijun Pu. On-line Real-Time Monitoring System for UHF Partial Discharge Prevention of Gas Insulated Switchgear Failure[J]. Power $\mathrm{Gr}$ id Technology,2016,09(14):366-368.

[2] Yonggang Guan, Jiali Qian. Application of Radio Frequency Method in Monitoring of High Voltage Switchgear Partial Discharge[J]. High Voltage Apparatus, 2015, 06(05): 181-183.

[3] Yunpeng Zhang, Huibin Wang, Juan Wang, et al. Research on UHF Online Monitoring System for Partial Discharge of High Voltage Switchgear[J]. High Voltage Apparatus, 2016, 11(10): 374-376.

[4] Xin Li, Chengrong Li, Yasha Li, Wei Wang, Hailiang Li. Analysis of GIS Partial Discharge Propagation by Finite Time Domain Difference Method[J]. Proceedings of the CSEE, 2015,03(17):283-285.

[5] Gang Chen. Application of Acoustic Wave Detector in Localization Detection of 10kV Switch Cabinet[J]. Electrical Engineering, 2014, 15(07): 315-318.

[6] Jieyi Luo. Research on Insulation State Evaluation Technology of 10kv Switchgear Based on Transient Ground Voltage and Ultrasonic Testing [D]. South China University of Technology, 2010.

[7] Pan Chen, Chenguo Yao, Ruijin Liao, Yi Chen, Yan Mi. Application of Sub-Band Energy Spectrum and Markov Clustering Algorithm in Partial Discharge Pattern Recognition of Switchgear[J]. High Voltage Technology,2015,09 (10): 390-392.

[8] Bell, Raymond, Charlson, Chris, Halliday, Shaun Paul, Irwin, Terry, Lopez-Roldan, Jose, Nixon, John. High-voltage on Site Commissioning Tests for Gas-Insulated Substations Using UHF Partial Discharge Detection. IEEE Transactions on Power Delivery. 2013. 
[9] Juan Wang. Research on On-Line Monitoring of Partial Discharge of High Voltage Switchgear Based on UHF [D]. North China Electric Power University (Hebei), 2015.

[10] Liangqi Si, Yong Qian, Wanjian Bai, Haifeng Ye, Yue Hu, Gegao Sheng, Xiuchen Jiang. GIS UHF Partial Discharge Pattern Recognition Based on Support Vector Machine[J]. High Voltage Apparatus,2014,37 (11): 403-405. 